\title{
Late onset atopic dermatitis and vitiligo: A case report
}

\author{
Sibel Berksoy Hayta, Rukiye Güner, Melih Akyol
}

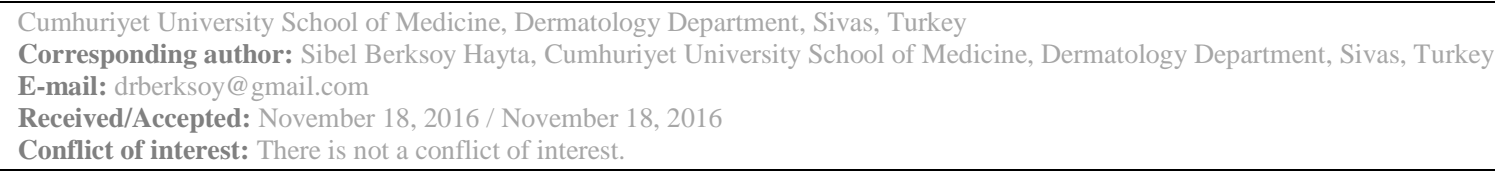

\section{Dear Editor,}

Adult-onset atopic dermatitis (AOAD) (onset $>18$ years) is a subgroup of atopic dermatitis (AD). This subgroup has been mentioned by Bannister and Freeman from Australia ${ }^{1}$. It may have different morphologic variants. Apart from the most typical flexural localization and eczematous pattern in adults, patients may also have a nonflexural distribution and other morphologic variants such as nummular or prurigo-like pattern ${ }^{2}$. The Hannifin and Rajka criteria can be used to diagnose $\mathrm{AOAD}^{3}$. $\mathrm{AD}$ is occasionally associated with vitiligo, and patients with vitiligo, especially early onset may appear to have increased risk of $\mathrm{AD}^{4}$.
We present a rare case with late onset atopic dermatitis and vitiligo. A 16 year old boy suffering from itchy lesions and dryness on his whole body, especially on flexural distributions for 6 months admitted to our out-patient policlinic. He also complained depigmented lesions. No personal history of atopy. His mother has an allergic rhinitis for many years.

Dermatologic examination of the patient revealed pruritic dry skin, pilar keratosis, and symmetric lichenified flexural eczema. $\mathrm{He}$ has a facial erythema, orbital darkness and Dennie-Morgan folds (Figure 1).

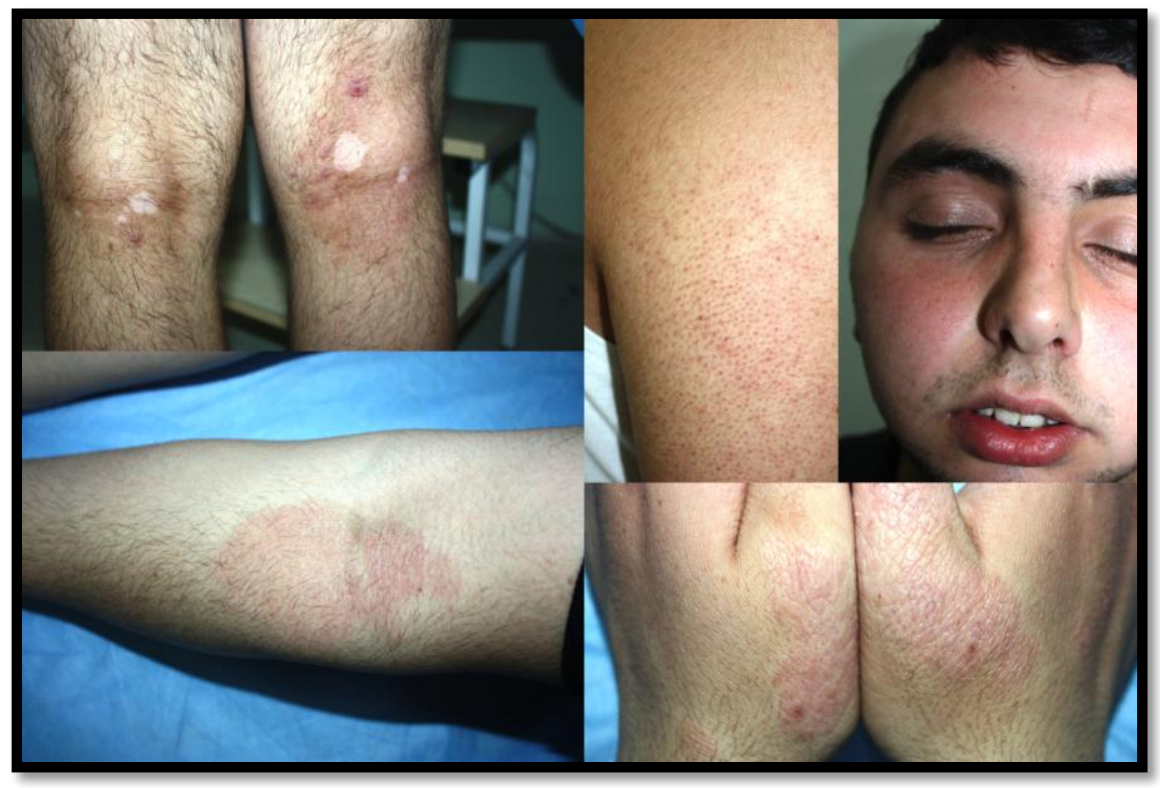

Figure 1. Clinical presentations of our case. 
Potassium hydroxide preperations were negative. Histopathologic examination of the lesions showed the findings of chronic dermatitis. The diagnosis of atopic dermatitis was made based on Hanifin-Rajka criteria.

He has also symmetric depigmented lesions on the popliteal skin area. Wood lamp examination of these lesions revealed blue-white light areas with sharp margins.

Haematologic and urine analysis, including IgE, ANA and antithyroid antibodies were in normal range.

Atopic dermatitis is a chronic or chronic relapsing, the classic morphology and location of which differs depending on age, and which is usually associated with severe pruritus. AD is a common skin disease, both in childhood and adolescence and in adults. The term AOAD (onset $>18$ years) was introduced by Bannister and Freeman ${ }^{1}$. The onset age of the disease in our case was 16 . Because of that, our case is not an AOAD. However, his diagnosis may be accepted as a late-onset atopic dermatitis.

There is lack of diagnostic criteria useful in both children and adults. Therefore understanding the natural history of AD is complicated. The UK working party criteria may not perform well among adults $^{6}$. Ozkaya ${ }^{1}$ reported that there is no problem in detecting early-onset $\mathrm{AD}$ according to The UK working criteria, but approximately one fourth of patients could not be given the diagnosis of AOAD according to this in its current form. The criteria of Hanifin and Rajka have been considered as a gold standard for $\mathrm{AD}$ diagnosis for the last 20 years, and this criteria was used in the diagnosis of our case. A diagnostic difficulty may arise when the onset occurs after the adolescence or later, as in these cases the clinical pattern may be atypical. However it may still present with flexural dermatitis ${ }^{3}$. Our case had a typical typical flexural distribution and lichenified eczematous pattern.

$\mathrm{AD}$ may be associated with specific subsets of vitiligo, and may indicate poor prognostic value for vitiligo. The mechanisms of association between $\mathrm{AD}$ and vitiligo are yet unknown. Patients with vitiligo, especially early onset, appear to have increased risk of $\mathrm{AD}^{4}$. The onset time was almost the same for both diseases in our case. However, while our case has a late onset time for $\mathrm{AD}$, but early onset for vitiligo.

Further studies are needed to explain the association between atopic dermatitis and vitiligo, and common disease mechanisms for both diseases.

\section{REFERENCES}

1- Bannister MJ, Freeman S. Adult-onset atopic dermatitis. Australas J Dermatol 2000; 41: 225-8.

2- Ozkaya E. Adult-onset atopic dermatitis. J Am Acad Dermatol 2005; 52: 579-82.

3- Kanwar AJ, Narang T. Adult-onset atopic dermatitis: under-recoginized or under-reported? Indian Dermatology Online Journal 2013; 4 16771.

4- Mohan GC, Siverberg J. Association of vitiligo and alopecia areata with atopic dermatitis: A systematic review and meta-analysis. JAMA Dermatol 2015; 151: 522-8.

5- Werfel T, Heratizadeh A, Aberer W, et al. S2k guideline on diagnosis and treatment of atopic dermatitis - short version. JDDG 2016; 14: 92-105.

6- Lan CC, Lee CH, Lu YW, et al. Prevalence of adult atopic dermatitis among nursing staff in a Taiwanese medical center: a pilot study on validation of diagnostic questionnaires. J Am Acad Dermatol 2009;61: 806-12. 